\title{
Destrutturare l'aula, ma con metodo: spazi e orizzonti epistemologici per una didattica aumentata dalle tecnologie ${ }^{*}$
}

\section{Paolo Ferri - Stefano Moriggi}

Università degli Studi di Milano-Bicocca - Department of Human Sciences (Italy)

doi: 10.7358/ecps-2016-013-ferr

paolo.ferri@unimib.it

stefano.moriggi@unimib.it

DECONSTRUCT THE CLASSROOM, BUT WITH METHOD:
SPACES AND EPISTEMOLOGICAL HORIZONS
FOR A DIDACTICS IMPLEMENTED BY TECHNOLOGIES

Abstract

This first paragraphs of this paper has been developed into two levels of argumentation. The first aimed to perform a genealogical analysis of the role of the different technologies (book and digital media) in redefine time, spaces, methods and relationships within the learning environments. More in detail we try: (a) to define the relationship between evolution of educational technologies and the new growing strategies for learning and teaching; (b) to understand the close connection (cultural and practical) among digital media, new teaching methods (based on the constructivist paradigm) and different educational environments and settings. As a consequence, the paper sketches an analysis of the "new» digitally augmented spaces for learning. In particular, we point out how methodological and didactical innovation and design of new "spaces and multiple environments» for learning are inextricably linked. They are actually the spaces in which it possile to apply new technologies for augmented learning. From all these points of view, the traditional classroom is definitely obsolete.

* I paragrafi 1 e 2 sono stati realizzati da S. Moriggi; i paragrafi 3 e 4 da P. Ferri. 
Keywords: Classroom, Cooperative learning, Flipped classroom, Learning spaces, Technology enhanced learning.

\section{INTRODUZIONE: UN «PASSO INDIETRO» PER GUARDARE AVANTI}

Una riflessione che voglia ragionare attorno alle forme e alle dinamiche che saranno proprie degli spazi di apprendimento compatibili con una ontologia aumentata dalle tecnologie richiede, paradossalmente, il «passo indietro» tipico della pratica filosofica (Heidegger, 1957). Ovvero, quella strategia teoretica che, per avviare una prudente e consapevole comprensione di ciò che ancora non sappiamo, (ri)chiede di mettere in discussione proprio ciò che invece abbiamo l'illusione di conoscere così bene, al punto da non ritenerlo meritevole di altra e ulteriore indagine. Tale atteggiamento trova una formulazione sintetica e radicale in un celebre passo della "Prefazione» alla Fenomenologia dello Spirito (1807) di Hegel - ove il filosofo di Stoccarda indica in questo approccio lo stile stesso dell'esercizio teoretico con queste parole: «Ciò che in generale è noto, proprio perché è noto, non è conosciuto". E non lo è perché - come spiega ancora Hegel - «il modo più consueto di ingannare se stessi e gli altri consiste appunto nel presupporre, nella conoscenza, qualcosa in quanto già noto, e nel farselo andare bene così com’è» (Hegel, 1986).

Pertanto, se l'ambizione del presente contributo è di comprendere (e per certi versi agevolare) le ragioni di una evoluzione culturale che starebbe per investire il mondo della scuola, dalle strategie didattiche alla riprogettazione degli spazi (reali e digitali), non ci si può esimere dal cominciare a mettere in discussione proprio quelle convinzioni e quelle consuetudini che nel tempo hanno plasmato le forme e i significati dei luoghi e dei metodi deputati all'insegnamento. E che, più o meno consapevolmente, sono intersoggettivamente ritenute le basi ovvie - per alcuni addirittura «naturali» - di un'istruzione e di una formazione plausibili.

Mettere in discussione, però, non significa semplicemente smantellare o, ancor peggio, archiviare la tradizione abbracciando ciecamente una qualche presunta innovazione. Al contrario, si tratta anzitutto di analizzare le vicende storiche e culturali che hanno reso possibile nei secoli la definizione e la diffusione di un certo modello di didattica e dei relativi spazi. Un'operazione, questa, che non può risolversi in un esercizio di memoria storica; ma che, invece, deve riuscire a intercettare le dinamiche sottese all'evoluzione di pratiche e interazioni in uno spazio deputato all'apprendimento. 


\section{GLI SPAZI DELL'APPRENDIMENTO: UNA ARCHEOLOGIA PER IL FUTURO}

Tra i molti spunti da cui si potrebbe cominciare un'indagine di questo tipo, si è deciso in questa sede di privilegiare un accostamento alla questione tanto diretta quanto empirica: entrare (o immaginare di entrare) in un'aula di una qualsiasi scuola italiana e cominciare a guardarsi attorno, osservando quel che accade.

Che sia in corso una lezione, un compito in classe o, magari, un'interrogazione, si potrebbe notare, per esempio, che i banchi in cui siedono gli studenti sono generalmente contrapposti alla cattedra da cui parla l'insegnante. Il che potrebbe sembrare ovvio: dopotutto, tale disposizione parrebbe agevolare la comunicazione tra il docente e i discenti, rendendo, più nello specifico, praticabile la lezione nei termini di una trasmissione di concetti e nozioni da uno (il docente) a molti (i discenti).

Sono proprio "ovvietà» di questo tipo quelle che dovremmo cominciare a interrogare per cercare di intuire come e perché tale rappresentazione dello spazio didattico è venuta a consolidarsi in un vero e proprio "canone» dell'insegnamento: nel senso proprio di quella abitudine acquisita come buona pratica della didattica. Tuttavia, per procedere effettivamente in tale direzione, occorre analizzare gli strumenti che hanno generato e modellato un dato contesto di relazioni e interazioni, qualificando uno spazio e precisando i ruoli degli attori in esso coinvolti.

Assumendo, pertanto, questo approccio di ricerca, lo strumento decisivo su cui focalizzare l'attenzione è il libro. Certo, concettualizzare il libro nei termini di uno strumento potrebbe anche suonare riduttivo; d'altra parte, se non ci si vuole condannare all'autoinganno e, al contrario, ci si prefigge di scalfire quell' "ovvietà scolastica» (l'aula tradizionale) che apparentemente non chiede ulteriore indagine, si tratta proprio di prendere sul serio il fatto che nessuno strumento - a partire dal libro - sia (e possa essere inteso) esclusivamente come un mezzo concepito e realizzato in vista di una serie di fini. Dello strumento bisognerà quindi cogliere la portata concettuale; così come, simmetricamente, di ogni concetto non dovrà sfuggire la ricaduta strumentale.

Assumendo, pertanto, questa prospettiva teoretica, per concentrarsi sul libro si rende necessario un secondo passo indietro, che si compirà avvalendosi di quanto sostenuto recentemente dal sociologo Michel Serres. «Da molto tempo e fino a poco fa - scrive lo studioso francese in un suo agile e provocatorio volumetto - il sapere aveva per supporto il corpo del sapiente, aedo o cantore del villaggio». Non si dava distinzione materiale (e tanto meno concettuale) tra il corpus della conoscenza e il corpo di chi la deteneva e la 
dispensava. Il sapere era il sapiente, al tempo dell'oralità. Ma «a poco a poco, il sapere si è oggettivato - continua Serres - prima nei rotoli, sui velini o sulle pergamene, supporti di scrittura; poi, a partire dal Rinascimento, nei libri di carta, supporti di stampa; infine oggi nel Web, supporto di messaggi e di informazioni» (Serres, 2012).

Seguendo questa linea, non si fatica a rileggere l'evoluzione dei supporti e dei dispositivi che hanno consentito di amplificare gli orizzonti della fruibilità e della condivisione delle conoscenze come un processo di progressiva esternalizzazione del sapere. Che, sempre e di nuovo, ha prodotto una riconfigurazione delle modalità di trasmissione e di comunicazione di contenuti, concetti e informazioni.

Ed è proprio all'interno di questa reiterata «alienazione» del corpus del sapere dal corpo del sapiente che dobbiamo ricollocare lo strumento-libro per rendere palese la ricaduta culturale della sua diffusione. Proprio a partire da un'aula scolastica.

Come è noto, dalla comparsa del primo artefatto tipografico partorito dalla stampa a caratteri mobili di Johannes Gensfleish zur Laden Gutenberg (autunno del 1455) alla produzione di testi scolastici «maneggiabili» da docenti e studenti dovette passare del tempo. Si consideri, infatti, che quella Bibbia prodotta dalla rivoluzionaria macchina dell'artigiano di Magonza misurava oltre quaranta centimetri di altezza e quasi trenta di larghezza. E che solo nel XVI secolo il libro cominciò lentamente ad assumere un aspetto più simile a quello che noi conosciamo.

"Le pagine si erano rimpicciolite, i margini ridotti e non comparivano più le glosse a margine. I cambiamenti - spiega il calligrafo Ewan Clayton avvennero in nome di una semplificazione tecnica dei libri, ma anche per assicurarne una sopravvivenza commerciale». Il tutto, però, nella consapevolezza che «un'impaginazione più essenziale, rendeva il libro più leggibile, consentendo una più facile articolazione degli argomenti all'interno del testo» (Clayton, 2013).

La continua ridefinizione del rapporto tra forma, struttura e funzione dell'oggetto-libro rispondeva così alle nuove leggi di una economia della cultura che, a sua volta, si andavano rimodellando in parallelo all'evoluzione e alla diffusione del libro medesimo. Scrive ancora Clayton: «Il testo, dapprima stampato in blocchi solidi, cominciò a essere suddiviso in paragrafi [...]. L'introduzione dell'indice fu poi la novità più significativa, perché inaugurò un nuovo modo di leggere» (Clayton, 2013). Ovvero, non solo dall'inizio alla fine del testo, ma anche procedendo a una consultazione mirata dei capitoli o dei paragrafi in cui trovare ciò di cui, di volta in volta, si era in cerca.

Non è questa la sede per ricostruire nel dettaglio vicende e fatti innescati dall'invenzione della stampa; tuttavia, a proposito di nuovi modi di 
leggere, è il caso quantomeno di ricordare che la produzione su larga scala della Bibbia in primis (e, a seguire, di altri testi), pose le condizioni materiali e culturali di quella lunga storia che Serres riassume precipita nella concretezza dell'aula scolastica nella quale ci siamo proposti di entrare. Ovvero, di quello spazio in cui l'iterato utilizzo dello strumento-libro ha visto delinearsi la figura di un «insegnante-sacerdote» che, avvalendosi delle sue competenze e delle sue abilità comunicative, dà voce ai contenuti di un testo nel tentativo farli arrivare nel modo più diretto e accessibile a una platea di «alunni-fedeli» i quali, a loro volta, cercheranno di assorbire la lezione, ripetendola a oltranza perché qualcosa rimanga impresso.

«Fino a questa mattina - scrive ancora Serres, commentando la genealogia dello spazio didattico tradizionale - un insegnante, in classe o in un'aula universitaria, dispensava un sapere che era già dispensato nei libri, almeno in parte. Trasmetteva oralmente uno scritto, una pagina-fonte. [...] La cattedra imponeva l'ascolto del portavoce del sapere. Per quell'oralità, chiedeva silenzio" (Serres, 2012).

Questa mattina, però, accanto al libro (e non al suo posto!) un nuovo - e ben più vasto - orizzonte di contenuti si sta affacciando sulla soglia dell'aula scolastica: il Web. Sovrabbondante ed eccessivo, sconfinato e contraddittorio, finirà per travolgere ogni portavoce del sapere che cercherà di opporsi al suo flusso. Provare a resistere, sarebbe ingenuo, oltre che vano. Perché significherebbe non aver capito quanto profondo e complesso è il nostro rapporto con le tecnologie, a cominciare proprio dal libro.

Sempre di più, dentro e fuori la scuola, sarà necessario pertanto saper scegliere: ovvero, essere in grado di distinguere in un contesto tanto vasto quanto inedito ciò che è affidabile, da ciò che non lo è; ciò che può essere utile da ciò che, invece, può solo sembrarlo. In altre parole, si tratterà di imparare a orientarsi in una realtà aumentata dall'ultima generazione di macchine che accompagna la nostra evoluzione culturale e che sempre più si è andata declinando in forme, per esempio i social network, che possono anche prestarsi a un uso distorto, inadeguato non produttivo. $\mathrm{Ma}$ - come insegnava Immanuel Kant già nel 1786 - orientarsi in uno spazio più o meno conosciuto (ed esperito) è una buona metafora per spiegare cosa significa pensare ${ }^{1}$.

Provare, dunque, a pensare l'aula (e più in generale la scuola) aumentata dalle tecnologie significa cercare di comprendere al meglio quali metodologie, dinamiche e relazioni (e dunque quali spazi, reali e virtuali) potranno

1 Si allude a qui al celebre testo di I. Kant, Cosa significa orientarsi nel pensiero (Kant, 1983), in particolare alle pp. 45-51. Per un approfondimento della metafora kantiana dell'orientamento calata in un contesto didattico aumentato dalle tecnologie sia lecito il rimando a Moriggi, $2014 \mathrm{~b}$. 
riconfigurare la totalità teorie e delle pratiche didattiche. Ma per fare questo, di nuovo, occorre iniziare con qualche breve considerazione attorno allo strumento in questione, il Web, e alle ricadute conseguenti alla sua diffusione.

Friedrich Engels sosteneva che, oltre un certo limite, la quantità si trasforma in qualità. Nel senso che, superato un certo livello, un incremento quantitativo genera un mutamento qualitativo nella natura stessa di un fenomeno. Tale considerazione del filosofo tedesco aiuta a ribadire come la pervasività del Web - per lo meno da quando il CERN di Ginevra decise (30 aprile 1993) di rendere pubblico il World Wide Web rinunciando a ogni diritto d'autore - abbia effettivamente definito un orizzonte qualitativamente diverso entro cui reimparare sempre e di nuovo a orientarsi, dentro e fuori l'aula scolastica.

Il mare magnum di dati e informazioni in cui da quel momento ci si è trovati a navigare configura un contesto di gran lunga differente da quello costituito da tutte le «mappe cartacee» che, fino a qualche decennio fa, ancora riuscivano a contenere (e a com-prendere) l'intero universo dei saperi e delle informazioni disponibili.

$\mathrm{Si}$ prospetta, pertanto, come un'urgenza culturale (prima ancora che didattica) quella di immaginare strategie di apprendimento, di trasmissione e di conservazione del sapere capaci di contenere i probabili rischi e ottimizzare le concrete opportunità di una (inedita) realtà aumentata.

Da questo punto di vista, la pregnanza della sopracitata metafora kantiana - quella per cui, appunto, l'esercizio del pensiero possa intendersi come una attività sotto molti aspetti analoga a quella dell'orientarsi in uno spazio più o meno conosciuto (ed esperito) - ritrova una sua inattesa attualità. Infatti, in un orizzonte tanto «aumentato", la pratica di un orientamento consapevole dovrebbe diventare parte costitutiva e fondante di un percorso didattico e formativo - da estendere dunque ai docenti, prima ancora che agli studenti; e finalizzato addirittura alla costruzione di un progetto di cittadinanza tecnico-scientifica.

In concreto, va qui delineandosi l'esigenza di imparare a pensare costruttivamente con le macchine che stanno riplasmando l'«ontologia quotidiana» entro cui andiamo riscrivendo il nostro modo di abitare il mondo (Moriggi, 2014a).

E per riuscirci in modo costruttivo, sarebbe molto d'aiuto cominciare (davvero!) a considerare cultura a tutti gli effetti anche la pratica scientifica. Se si iniziasse, infatti, a contemplare più da vicino (e con qualche pregiudizio in meno) il lavoro artigianale dello scienziato ${ }^{2}$, ci si renderebbe conto di co-

2 Il termine «artigiano» rievoca qui implicitamente la riflessione sviluppata da Richard Sennett nel suo L'uomo artigiano (Sennett, 2008). 
me anche lui - e per lo meno dai tempi di Galilei - sia costretto quotidianamente a ri-orientarsi in un denso flusso di dati e di informazioni che confluisce in un orizzonte davvero sconfinato: quello, appunto, della sua ignoranza.

Utilizzare e perfezionare strumenti (concreti e concettuali) per gestire razionalmente la propria ignoranza è uno degli elementi decisivi che consentono di reperire nella pratica scientifica la matrice culturale per una concreta e rigorosa attivazione di competenze utili a vivere più criticamente il portato della rivoluzione tecnologica cui stiamo assistendo.

E questo proprio perché in un mondo - o in un'aula - in cui si teme (per certi versi a ragione) che l'abbondanza rischi di sopraffarci, è importante riuscire a distinguere e scegliere con criterio e autonomia di giudizio. In altre parole, appunto, saper orientarsi, kantianamente.

Ma l'importanza della pratica scientifica - o per meglio dire, della logica della scoperta scientifica - quale matrice culturale di un progetto formativo tecnologicamente aumentato può anche utilmente essere evidenziata recuperando, ancorché per brevi cenni, il contesto in cui è maturata l'invenzione del Web.

Come noto, teatro degli eventi è stato il CERN di Ginevra. Correva l'anno 1984 e Timothy (Tim) Berners-Lee, vinta una borsa di studio, viene assegnato all'équipe che si occupa di raccogliere e classificare i risultati degli esperimenti realizzati in tutto l'istituto di ricerca. Il giovane ricercatore fin da subito intese che il suo progetto informatico avrebbe dovuto non solo custodire e ordinare un patrimonio di dati ed evidenze scientifiche destinato a crescere di giorno in giorno; ma addirittura "aumentare» il livello di cooperazione di quella numerosa e complessa comunità di ricerca.

Un modus operandi che, a ben vedere, finiva con il riprodurre nella quotidianità di una pratica collaborativa alcune delle caratteristiche tipiche e costitutive del sapere scientifico moderno in quanto tale. Ovvero, il fatto di essere - e per la prima volta nella storia - un sapere pubblico, rivedibile e controllabile: rivedibile in quanto controllabile e controllabile perché pubblico.

Pertanto, se analizzando più da vicino la storia prima di Internet e poi del Web, emerge piuttosto chiaramente che lo strumento in questione incarna e aumenta le dinamiche interne che regolano e scandiscono la critica e la crescita della conoscenza scientifica; parrebbe quanto meno ingenuo un progetto formativo e didattico che volesse implementare un setting d'aula con quello stesso strumento, trascurando però la matrice culturale che l'ha reso in prima istanza possibile e in seguito necessario.

In altre parole, nella misura in cui il Web giungerà ad aumentare la didattica, proporzionalmente si tratterà di sviluppare modelli e spazi per l'apprendimento in grado di riprodurre quanto possibile e quanto necessario le strategie di apprendimento proprie di una comunità di ricerca. 


\section{AMBIENTI FLESSIBILI E SPAZI COOPERATIVI}

L'«aumento» digitale degli spazi della scuola e il ribaltamento del setting didattico che abbiamo sopra evocato, come una possibile via per l'uso significativo delle tecnologie nella didattica, a nostro avviso, implica un ripensamento più generale dell'organizzazione scolastica che, appunto, non trascuri gli spazi fisici e le unità architettoniche, come del resto auspicano anche una serie di studi e di ricerche condotti dall'OCSE ${ }^{3}$.

Si prenda, per esempio, il caso del cooperative learning, che può costituire una delle premesse teoriche, di una appliacazione "significativa" della didattica digitale. Il cooperative learning prevede, l'accentuazione della collaborazione fra studenti, l'incremento dello scambio di conoscenze e dell'aiuto reciproco, tutte istanze che spingono verso la progettazione di una scuola che tenga nel dovuto conto la vita sociale degli alunni e il modo in cui essa può essere messa al servizio dell'apprendimento. Alle realizzazione di questo obiettivo possono concorrere in maniera rilevante le potenzialità comunicative, sociali e interattive delle comunicazioni digitali. La necessità di una maggior «socialità» della didattica e una sua più stretta correlazione con le pratiche informali della vita sociale degli alunni - sempre più spesso mediata da strumenti di natura digitale (Jenkins, 2009) - non può essere, ovviamente, limitata entro mura dell'aula. E tuttavia anche dentro le mura dell'aula questa tendenza dovrebbe venire assecondata (e non repressa) dagli spazi stessi.

La metodologia della flipped classroom o della "classe ribaltata" (Bergmann \& Sams, 2009, 2014a, 2014b) ${ }^{4}$ - come quella del cooperative learning

3 A questo proposito si veda in particolare la base dati tematica dell'OCDE, Architecture, planning, design, disponibile all'interno del portale dell'OCDE al sito: http://www.oecd. org/edu/innovation-education/architectureplanninganddesign.htm; oltre al portale "privato» Designshare Designing for the future of learning che raccoglie miglia di risorse Web su questo stesso tema (http://www.designshare.com/index.php/home). Inoltre, per un panorama sull'innovazione dell'edilizia scolastica in Italia si può consultare la base dati di INDIRE: Quando lo spazio insegna (http://www.indire.it/quandolospazioinsegna/).

4 La flipped classroom, o classe ribaltata, è un sistema che, attraverso l'uso delle tecnologie didattiche, inverte il tradizionale schema di insegnamento/apprendimento e il conseguente rapporto docente/discente. I materiali didattici vengono caricati all'interno dell'ambiente virtuale per l'apprendimento (alcuni anni fa si chiamava piattaforma di elearning) del "gruppo classe» in forme e linguaggi digitali anche molto differenziati. Per approfondire un contenuto o un tema non si utilizzano più solo testi scritti, ma anche audio, video, simulazioni e materiali disponibili su Internet. Questi materiali possono essere approfonditi dagli studenti da soli o in gruppo "fuori dalla classe»: a casa, in biblioteca o in altri luoghi di aggregazione informale. Mentre in classe, con l'insegnante, i contenuti «appresi» attraverso la tecnologia diventano oggetto di attività cooperative mirate a «mettere in movimento» le conoscenze acquisite. La classe non è più dunque il luogo di trasmissione delle nozioni, ma piuttosto lo spazio di lavoro e di discussione dove si impara a utilizzarle nel confronto con i pari e con il docente. Su questo tema 
del resto - richiedono spazi modulari e poli-funzionali, facilmente riconfigurabili, oltre che in grado di rispondere a metodologie didattiche e progetti formativi innovativi. Progetti, però, incentrati sull'attivazione delle conoscenze e sulla risoluzione di problemi; e non più sul nozionismo e sullo studio individuale. Se, infatti, si analizza la distribuzione degli spazi delle «nuove scuole» che vengono costruite (contrariamente a quanto avviene nella maggior parte delle scuole italiane ${ }^{5}$ ) in alcuni paesi europei ${ }^{6}$, ci si rende conto di come si possano individuare due costanti comuni a tutti quegli edifici:

a. Una pervasiva, ma «invisibile», infrastrutturazione tecnologica: banda larga, hot spot wireless, ambienti virtuali per la gestione della didattica (virtual learning environment), tablet/notebook per studenti e insegnanti.

b. L'assenza dell'aula come unità minima architettonica e metodologicodidattica della scuola. L'aula tradizionale è troppo grande per essere un "laboratorio", ma troppo piccola per essere un auditorium!

La struttura di base degli spazi di una scuola "aumentata» dalla tecnologia, l'aula, è sostituita così da una varietà di nuove tipologie di ambienti e spazi (reali e digitali) - inclusi, ovviamente, gli «ambienti virtuali per l'apprendimento». La spazio architettonico e didattico più diffuso nelle nuove scuole è pertanto proprio quello del "laboratorio" dove si svolgono quelle "attività» che prendono in gran parte il posto delle lezioni tradizionali (e che, ovviamente, sono accompagnate dal supporto e dal tutoring degli insegnanti). Ogni scuola ha poi spazi comuni per l'apprendimento informale e per il peer tutoring abilitato dalle tecnologie, oltre a specifiche aree "protette" per lo studio individuale e a un auditorium ove i docenti più preparati e competenti (oppure esperti esterni) possono tenere lectures sui «nuclei fondanti» delle varie discipline a tutti gli studenti dello stesso anno (Barrett \& Zhang, 2009; Ferri, 2013). L'architettura, pertanto, segue la metodologia: non più classi dove gli insegnanti della stessa disciplina, più o meno contemporaneamente, tengono

sia lecito il rimando a Ferri, 2013, cap. 3, e Ferri \& Moriggi, 2014).

5 Ovviamente, anche in Italia esistono esempi di scuole di eccellenza nell'ambito della didattica aumentata dalle tecnologie, quali: i nidi e le scuole della Fondazione Reggio Children (http://www.reggiochildren.it); l'Istituto Comprensivo Statale di Cadeo e Pontenure (http://www.istitutocomprensivocadeo.it); l'IISS Ettore Majorana di Brindisi (http://www. majoranabrindisi.it); l'ITTS Alessandro Volta di Perugia (http://www.avolta.pg.it), oltre alle scuole che fanno parte del progetto Avanguardie Educative promosso dall'INDIRE (http:// www.avanguardieeducative.indire.it). Tuttavia, fino a oggi queste e altri contesti di eccellenza, sparsi un po' su tutto il territorio nazionale, rappresentano ottime pratiche ed esperienze che, però, ancora non riescono a fare massa critica e ad esportare l'innovazione su tutto il sistema nazionale dell'istruzione.

$6 \mathrm{Si}$ allude qui a paesi che investono più del nostro in educazione, come, per esempio, gli USA o altri Stati del Nord Europa. L'Italia, secondo i dati OCSE, è buona penultima per quanto concerne gli investimenti nel sistema formativo; ed è seguita solo dalla Grecia (OCDE, 2014). 
lezioni frontali e nozionistiche sugli stessi contenuti a 25/30 allievi alla volta; ma lectio magistralis su nuclei fondanti rivolte a tutti gli studenti di uno stesso anno, oltre a laboratori per sviluppare in progetti concreti le nozioni operativamente apprese.

La tradizionale didattica d'aula quindi - come si è visto anche più sopra - evolve progressivamente in quella che si svolge (e non si può che svolgere) negli spazi laboratoriali, fisici e/o digitali, dove gli studenti, suddivisi in gruppi e sottogruppi (seguiti da uno o più insegnanti), sviluppano progetti e attività creative. Negli spazi di aggregazione informale, inoltre, gli allievi proseguono autonomamente i lavori in corso. Tali attività, proprio grazie all'ambiente virtuale di apprendimento, possono proseguire oltre l'aula, sempre con il tutoraggio (on-line) dell'insegnante.

Ma approfondiamo ulteriormente alcune delle premesse culturali e operative di questa "destrutturazione» dell'aula tradizionale. In primo luogo, è necessario prendere in considerazione il fatto che, nella costruzione di un setting didattico funzionale alle nuove esigenze di apprendimento dei «nuovi bambini» (Ferri, 2014), gli spazi fisici dell'apprendimento sono altrettanto importanti di quelli digitali (Internet e i software; cfr. Ferri, 2011).

In Italia, da anni, è difficile che vengano costruite nuove scuole: il calo demografico e la scarsità di investimenti in educazione hanno fatto uscire per molti anni questo tema dall'agenda della discussione pubblica - al netto dei recenti interventi normativi che paiono cominciare a invertire la rotta ${ }^{7}$. In altri paesi, invece, come gli USA, ma anche in Olanda, in Danimarca, o in Svezia - nonostante il calo demografico - si continuano a costruire edifici scolastici. E sono «scuole nuove»! Ovvero, progettate secondo criteri radicalmente diversi da quelli tradizionali (Barrett \& Zhang, 2009; Robinson \& Robinson, 2009; Rigolon, 2010; Nair, Fielding, \& Lackeny, 2013). Per esempio, Harel Copperton sostiene, proprio in questa direzione, che la scuola del XXI secolo non possa più avere come obiettivo strategico i tre postulali della didattica tradizionale: leggere, scrivere e far di conto. Ma, piuttosto, debba porsi traguardi più ambiziosi: l'esplorazione - ovvero, la capacità degli studenti (tutorati dai docenti con funzione di coach) di esplorare le nuove risorse digitali finalizzate all'apprendimento attivo e alla gestione attiva dei contenuti appresi per la risoluzione di problemi teorici e pratici; l'espressione - ovvero, la capacità di utilizzare media digitali (ma non solo) per esprimere idee e rappresentare conoscenze. Una capacità, questa, fondamentale in un mondo in cui la creati-

7 Ci si riferisce qui in particolare alla Legge 107/2015 - nota anche come «La Buona Scuola» (https://labuonascuola.gov.it/) - oltre al Piano Nazionale Scuola Digitale dell'ottobre del 2015 (http://www.istruzione.it/scuola_digitale/allegati/Materiali/pnsd-layout-30.10WEB.pdf). 
vità ha sostituito molte altre competenze in relazione alla capacità di generare valore sociale e individuale (Florida, 2002 e 2005). E la cooperazione - ovvero, l'abilità e la competenza relazionale, decisiva per i futuri cittadini di una società informazionale, di scambiarsi e di condividere conoscenze e idee ${ }^{8}$.

Per avviare questo tipo di genealogia delle competenze, si rendono pertanto necessari spazi fisici (oltre che digitali) compatibili con le metodologie attive che abbiamo più volte descritto (Ferri, 2013; Ferri \& Moriggi, 2014; Moriggi, 2015) ${ }^{9}$. Se si analizza, per esempio, la pianta della scuola Vittra di Stoccolma (Figura 1 - come anche le immagini dei suoi ambienti didattici http://www.rosanbosch.com/en/project/vittra-school-telefonplan), ci si può render conto di cosa significhi la trasformazione (anche) fisica degli spazi della didattica abilitata dalle nuove tecnologie. Osservando, per esempio, la pianta e tenendo altresì conto che la scuola Vittra è il corrispettivo di una primaria italiana (6-11 anni), si può notare che nella progettazione è venuto del tutto meno il consueto modello di aule allineate e lunghi corridoi. Il tutto è stato riconcettualizzato nei termini di un ambiente funzionale alle attività che, di volta in volta, i bambini si trovano a svolgere insieme e con i loro insegnanti.

Tali «spazi funzionali» sono stati attrezzati sulla base teorica degli studi condotti da Nair, Finley e Lackney (2013). Si tratta di spazi digitalmente e analogicamente infra-strutturati per rispondere alle diversificate esigenze degli studenti (CELE, 2011) Gli ambienti modulari e polifunzionali che osserviamo nella pianta, sono inoltre compatibili con lo sviluppo e la progettazione di una didattica personalizzata e ribaltata che utilizzi le metodiche più avanzate dell'attivismo-costruzionista. Tali metodologie presuppongono un'idea di insegnamento/apprendimento (Papert, 1980 e 1993; Gardner, 1983; Bruner, 1990; Brown \& Campione, 1994; Chiosso, 2003 e 2008; Jonassen, 2005; Campbell et al., 2007; Gee, 2007; Hartley, 2007; Pullen \& Cole, 2009; Ferri, 2014) che mette in valore il carattere attivo dell'apprendimento, la socialità e l'interattività dei soggetti coinvolti nei processi formativi e dei contesti educativi. Un'idea che negli ultimi due decenni ha trovato applicazione sempre più ampia, per esempio, nel Regno Unito; ma più in generale in tutto il Centro-Nord Europa.

8 Sul modo di tradurre in attività in classe questo nuovo approccio all'apprendimento si veda in particolare la metodologia delle e-tivities di Salmon (2002).

9 Nel merito della trasformazione degli spazi reali dell'apprendimento è molto rilevante il contributo di Nair, Finley e Lackney nel loro The language of school design: Design patterns for 21st century schools ( $1^{\text {a }}$ ed., 2005). Si tratta di un lavoro pionieristico che definisce un nuovo vocabolario grafico in grado di sintetizzare l'apprendimento attivo con le migliori pratiche della pianificazione scolastica e del design. Si ricorda anche su questo tema il volume di Harrison e Hutton, Design for the changing educational landsacape. Space, palce and the future of learning (2014). 


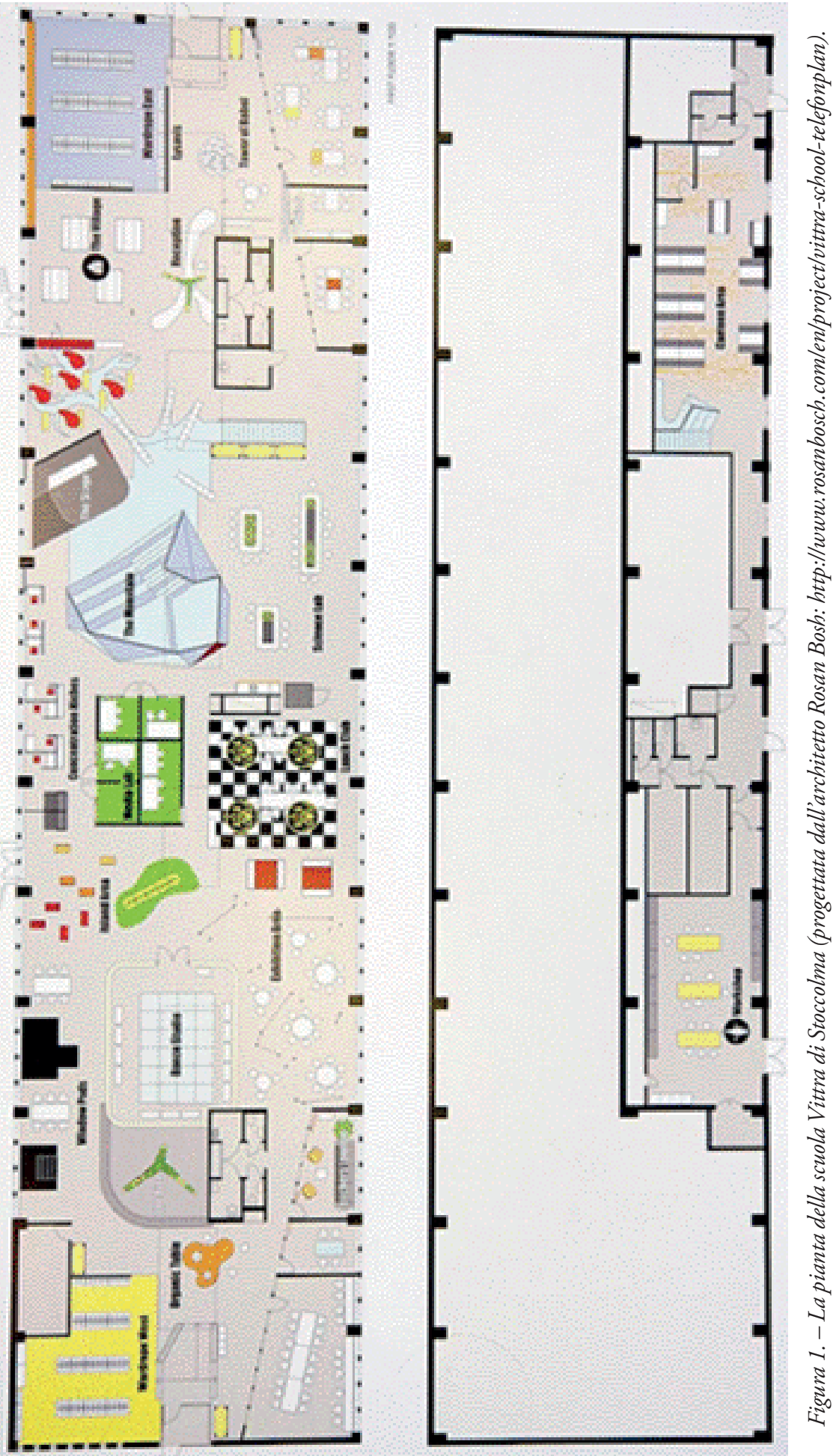


Si tratta, pertanto, di progettare e realizzare contesti formativi reali e digitali che costituiscano vere e proprie comunità di apprendimento - noi diremmo «laboratori di ricerca» - tecnologicamente aumentate e composte da insegnanti, studenti oltre che da genitori. Comunità di apprendimento modellate sui seguenti principi: (a) strategie efficaci di insegnamento e di apprendimento in rapporto alle disposizioni e alle inclinazioni degli allievi; (b) impostazione curricolare flessibile e di impronta metodologica; (c) valutazione di processo impostata in funzione dell'ulteriore apprendimento; (d) organizzazione scolastica comunitaria; (e) creazione di partnership significative per la vita scolastica con altre agenzie educative e sociali, ma anche imprenditoriali e produttive (Hartley, 2007; Mincu, 2008).

\section{UNA DIDATTICA A DIMENSIONI MULTIPLE}

Ora, combinando tale assetto teorico con l'orizzonte metodologico qualitativamente tratteggiato nel paragrafo 2 , è possibile dunque procedere a una trasformazione degli spazi della scuola che sia metodologicamente fondata.

Possiamo perciò con Nair, Fielding e Nackley auspicare la necessità di una scuola senza classi - o che per lo meno superi culturalmente il concetto di aula tradizionale. $\mathrm{E}$ che, dunque, preveda quanto meno i cinque ambienti di apprendimento schematizzati in Figura 2 (già realizzati in una serie di scuole innovative, tra cui, appunto, la sopracitata scuola Vittra).

Spazio cardine nell'economia didattica di questo elenco è, appunto, il «laboratorio». Tale ambiente si propone, infatti, come un luogo polifunzionale centrale nella prassi didattica quotidiana, in cui è possibile recuperare la dimensione manipolativo-esperienziale dell'apprendimento anche in chiave virtuale attraverso gli strumenti digitali. L'apprendere attraverso il fare è fondamentale in quest'ottica: è necessario pertanto che le «nuove scuole» abilitate dalle tecnologie contemplino ambienti laboratoriali ampi e ben attrezzati, specifici per le singole discipline.

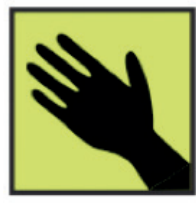

LABORATORY

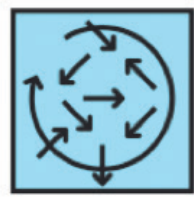

WATERING HOLE

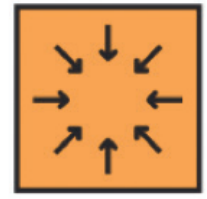

CAMPFIRE

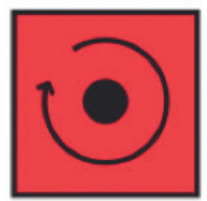

CAVE

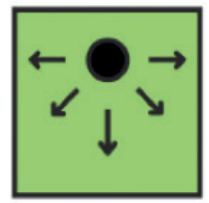

SHOW-OFF

Figura 2. - Una schematizzazione dei nuovi spazi dell'apprendimento teorizzati da Nair, Fielding, \& Nackley, 2013 (3a ed. rivista). 
La didattica frontale, come si diceva, in questo contesto diviene quindi solo una - spesso marginale - tra le modalità di attuazione dell'azione formativa. In particolare, all'interno di una scuola digitalmente aumentata, le capacità di simulazione delle "macchine digitali» favorisce (a) le modalità di didattica laboratoriali, (b) l'evoluzione della classe in una comunità di ricer$\mathrm{ca}$ - ossia, pratiche e approcci difficilmente realizzabili in modalità analogica.

Oltre ai laboratori, come accennato, si rendono necessari almeno altre quattro tipologie di ambiente didattico:

a. Aree di ritrovo informale. Sono appositi spazi di socializzazione tra bambini e studenti, che agevolano la comunicazione sociale e consentono alla didattica di proseguire anche al di là delle ore curricolari. La «cultura informale» dei «nuovi bambini» (Ferri, 2014) è strutturalmente crossmediale (Jenkins, 2009), per questo lo scambio di socialità e comunicazione, anche formativa, integra sia modalità in presenza sia modalità on-line.

b. Aree attrezzate per la discussione di gruppo. Considerando la dialettica interna alla comunità scientifica (e costitutiva del sapere scientifico moderno) come una matrice culturale imprescindibile della nostra proposta metodologica, diventa di conseguenza necessario prevedere nelle scuole ambienti predisposti per la discussione argomentata delle credenze e delle ipotesi di ricerca in gioco. Tali ambienti possono essere paragonati a «sale riunioni» aumentate digitalmente (dotate quindi di videoproiettore interattivo, device per la connessione wifi di tablet e computer allo strumento di proiezione) dove gli studenti tra pari e/o coordinati dal docente conducono sessioni di brainstorming e di (meta)discussione sui risultati provvisori o conclusivi delle loro attività.

c. Area per lo studio individuale. Anche in una scuola aumentata e cooperativa, la riflessione e lo studio individuale conservano un ruolo decisivo nell'economia didattica. Pertanto, è necessario prevedere un contesto accogliente e protetto - solitamente denominato cave (caverna) - dove, appunto, gli studenti possano in autonomia riflettere, leggere e dedicarsi a diverse attività individuali. La "caverna» nasce quindi per venire in contro agli stili e ai bisogni differenti e personali dei bambini e degli studenti; ma è anche un luogo importante dove svolgere attività in cui si richiede concentrazione e tranquillità - come, per esempio, lo studio di un libro, l'analisi di un articolo (anche su tablet), il montaggio di un video o il mixaggio di un file audio, ecc.

d. Esiste inoltre la necessità - secondo il modello che si è prospettato - di poter usufruire di un auditorium dove presentare i risultati del lavoro di ricerca da parte degli studenti. Luogo dove anche i docenti interni ed esterni possano - come si accennava più sopra - tenere lezioni e con- 
ferenze di approfondimento su temi specifici e di interesse per tutti gli allievi che frequentano, poniamo, un medesimo corso (inter) disciplinare. Si tratta del cosiddetto "teatro" (show-off): uno spazio poli-funzionale sicuramente adatto allo svolgimento di conferenze (o di attività comuni a più gruppi di lavoro), ma soprattutto utile al fine di condividere e valutare insieme il risultato dei progetti di gruppo. È il luogo dove può e deve avvenire la revisione razionale e la discussione sugli elaborati finali, anch'essi di natura cross-mediale e multi-codicale - per esempio, $b l o g$, video, composizioni musicali, ma anche presentazioni o ipertesti di approfondimento. Inoltre lo show-off può altresì recuperare la sua funzione originaria, diventando a tutti gli effetti un "teatro" in cui si possono guardare insieme video o film, piuttosto che mettere in scena rappresentazioni o drammatizzare attività svolte nei singoli ambiti disciplinari.

Questa nuova distribuzione degli spazi è plausibile, ovviamente, solo dove sia possibile costruire edifici ex novo. Tuttavia, anche in quei contesti in cui (come, per esempio, in Italia), al netto di fortunate eccezioni, non sia ancora possibile progettare ed erigere da zero un edificio scolastico, è tanto auspicabile quanto possibile che la stessa filosofia degli spazi venga precipitata nelle realtà preesistenti.

La configurazione ottimale per questo tipo di «adattamento progettuale» è la «ristrutturazione» dell'aula tradizionale, quanto più possibile, in uno spazio polifunzionale, dotato di banchi mobili e attrezzato con una postazione di controllo e gestione del processo e dell'organizzazione della didattica (che può essere un pc fisso, un pc portatile o anche un tablet connesso in cloud col sistema di software della scuola).

Lo schermo interattivo permetterà al docente di aprire l'aula all'esterno attraverso una connessione a Internet; così come di sfruttare il sistema cloud della scuola per gestire on-line una serie di procedure amministrative. Questi tipo di sistema-aula a geometria variabile sarà tanto più efficiente, quanto più verrà «specializzato" per disciplina. Se, infatti, non si potranno «abbattere i muri», sarà sempre più necessario metabolizzare l'idea che possano e debbano esistere classi-laboratorio disciplinari e che siano gli studenti - e non i docenti - a doversi spostare da un ambiente all'altro, secondo il calendario delle lezioni.

Anche questa soluzione «intermedia» - ma forse più realistica per il caso italiano - implica e richiede la medesima filosofia degli spazi didattici che come si diceva - conduce a un oltrepassamento (concettuale e fisico) della idea stessa di aula. 


\section{CONCLUSIONI}

L'insieme delle considerazioni che abbiamo sviluppato delinea un possibile scenario di progressiva trasformazione degli spazi della didattica e dell'apprendimento, che si propone come una sorta ideale regolativo di medio-lungo periodo. Siamo consapevoli, infatti, che il sistema formativo italiano solo recentemente (Legge 107/2015 e Piano Nazionale Scuola Digitale, Ottobre 2015) ha ripreso a investire sull'innovazione e sulle persone. Non a caso, nell'ultima parte del nostro intervento ci siamo soffermati su soluzioni che tenessero conto sia dei contributi internazionali più avanzati sia delle condizioni materiali della scuola italiana. Resta, in ogni caso, l'urgenza e la necessità di dare corpo a un ripensamento graduale ma fattivo degli spazi e dei metodi della didattica che sia coerente e conseguente con le sfide culturali ed epistemologiche che la nuova modalità digitale della trasmissione dei saperi ci pone di fronte.

\section{RIFERIMENTI BIBLIOGRAFICI}

Barrett, P., \& Zhang, Y. (2009). Optimal learning spaces design implications for primary schools. Salford, UK: Design and Print Group - University of Salford. http://www.oecd.org/edu/innovation-education/ centreforeffectivelearningenvironmentscele/43834191.pdf

Bergmann, J., \& Sams, A. (2012). Flip your classroom: Reach every student in every class every day. Washington: ISTE \& ASCD.

Bergmann, J., \& Sams, A. (2014a). Flipped learning: Gateway to student engagement. Washington: ISTE.

Bergmann, J., \& Sams, A. (2014b). Flipped learning-maximizing face time. Training \& Development, 68(2), 28-31.

Brown, A. L., \& Campione J. C. (1994). Guided discovery in a community of learners. In K. Mc Gilly (Ed.), Classroom lesson: Integrating cognitive theory and classroom practice (pp. 229-270). Cambridge, MA: MIT Press - Bradford Book.

Bruner, J. (1990). Acts of meaning. Harvard, MA: Harvard Univeristy Press (trad. it., La ricerca del significato. Torino: Bollati Boringhieri, 1992).

CELE (2011). Designing for education. Compendium of educational facilities. Paris: OECD. http://www.oecd.org/edu/innovation-education/ centreforeffectiveleningenvironmentscele/49057666.pdf

Campbell, R.J., Robinson, W., Neelands, J., Hewston, R., \& Mazzoli, L. (2007). Personalised learning: Ambiguities in theory and practice. British Journal of Educational Studies, 55(2), 135-154. 
Chiosso, G. (2008). Personalizzare l'insegnamento. Bologna: il Mulino.

Chiosso, G. (2003). Personalizzazione. In G. Cerini \& M. Spinosi (a cura di), Voci della scuola. Napoli: Tecnodid.

Clayton, E. (2013). The golden thread. The story of writing. London: Atlantic Books (trad. it., Il filo d'oro. Storia della scrittura. Torino: Bollati Boringhieri, 2014).

Ferri, P. (2011). Nativi digitali. Milano: Bruno Mondadori.

Ferri, P. (2013). La scuola 2.0. Verso una didattica aumentata dalle tecnologie. Parma: Spaggiari.

Ferri, P., \& Moriggi, S. (2014). La classe di Bayes: note metodologiche, epistemologiche e operative per una reale digitalizzazione della didattica nella scuola italiana. Educational, Cultural and Psychological Studies, 5, 1-18.

Feynman, R. P. (1999). The pleasure of finding things out. New York: Basic Books (trad. it., Il piacere di scoprire. Milano: Adelphi, 2002).

Florida, R. (2002). The rise of the creative class: And how it's transforming work, leisure and everyday life. New York: Basic Books (trad. it., L'ascesa della nuova classe creativa. Stile di vita, valori e professioni. Milano: Mondadori, 2003).

Florida, R. (2005). The flight of the creative class. The new global competition for talent. New York: Harper Collins (trad. it., La classe creativa spicca il volo. La fuga dei cervelli: chi vince e chi perde. Milano: Mondadori, 2006).

Gardner, H. (1983). Frames of mind: The theory of multiple intelligences. New York: Basic Books (trad. it., Formae mentis. Saggio sulla pluralità delle intelligenze. Milano: Feltrinelli, 1987).

Gee, J. P. (2007). What video games have to teach us about learning and literacy, revised and updated. Basingstoke: Palgrave Macmillan (trad. it., Come un videogioco. Insegnare e apprendere nella scuola digitale. Milano: Raffaello Cortina, 2013).

Harrison, H., \& Hutton, L. (2014). Design for the changing educational landsacape. Space, palce and the future of learning. London: Routeledge.

Hartley, D. (2007). Personalisation: The emerging 'revised' code of education. In Oxford Review of Education, 33(5, November), 629-642.

Hegel, G. W. F. (1986). Phänomenologie des Geistes. Frankfurt: Surkamp Verlag (trad. it., Fenomenologia dello spirito. Ed. it. a cura di G. Garelli. Torino: Einaudi, 1986).

Heidegger, M. (1957). Identität und Differenz. Pfullingen: Verlag Günther Neske (trad. it., Identità e differenza, Parte II: La concezione onto-teo-logica della metafisica. In Teoresi, 1967, 215-235).

Jenkins, H. (2009). Confronting the challenges of participatory culture. Media Education or the 21 st century. Cambridge, MA: MIT Press (trad. it., Culture partecipative e competenze digitali. Milano: Guerini \& Associati, 2013).

Jonassen, D. H. (2005). Modeling with technology: Mindtools for conceptual change (3rd ed.). Columbus, OH: Prentice-Hall. 
OECD (2014). Education at glance - OCDE indicators 2014. Paris: OECD - Centre for Educational Research and Innovation.

Kant, I. (1983). Was Heisst Sich im Denken Orientieren. In Werke in Sechs Bänden (pp. 265-283). Hrsg. von W. Weischedel, Band III. Darmstadt: Wissenschaftliche Buchgesellschaft (trad. it., Cosa significa orientarsi nel pensiero. Ed. it. a cura di F. Volpi. Milano: Adelphi, 1996).

Mincu, M. E. (2008). Fondazione per la Scuola. La personalizzazione, una scommessa per il futuro. Rassegna dell'Istruzione 1-2, Settembre/Ottobre - Novembre/ Dicembre 2009.

Moriggi, S. (2014a). Connessi. Beati quelli che sapranno pensare con le macchine. Cinisello Balsamo: Edizioni San Paolo.

Moriggi, S. (2014b). 'Che storia!' La metodologia della scienza come nuovo orizzonte della didattica e della cittadinanza. Novecento.org, 3, doi: 10.12977/ nov55.

Moriggi, S. (2015). Oltre gli idoli del senso comune. Per una educazione (scientifica) al digitale. Mediascapes Journal, 5, 62-70. http://ojs.uniroma1.it/index.php/ mediascapes/article/view/13242/13042

Nair, P., Fielding, R., \& Lackney, C. (2013). The language of school design: Design patterns for 21st century schools (3rd ed. revised). Salford, UK: Design and Print Group - University of Salford.

Papert, S. (1980). Mindstorms: Children, computers, and powerful ideas. New York: Basic Books (trad. it., Mindstorms: bambini, computer e creatività. Milano: Emme, 1984).

Papert, S. (1993). The children's machine: Rethinking school in the age of the computer. New York: Basic Books (trad. it., I bambini e il computer. A cura di A. Bellomi. Milano: Rizzoli, 1994).

Pullen, D. L., \& Cole, D. R. (2009). Multiliteracies and technology enhanced education. Social practice and the global classroom. Herschey, PA: IGI Group.

Rigolon, A. (2010). European design types for 21st century schools: An overview. Salford, UK: Design and Print Group - University of Salford. http://www.oecd. org/edu/innovation-education/centreforeffectivelearningenvironmentscele/ 43834191.pdf

Robinson, L., \& Robinson, T. (2009). An Australian approach to school design. CELE \& Exchange, 3. http://www.oecd.org/education/innovation-education/ centreforeffectivelearningenvironmentscele/42168991.pdf

Salmon, G., (2002). E-tivities: The key to active online learning. London: Kogan Page.

Sennett, R. (2008). The craftsman. New Haven, CT: Yale University Press (trad. it., L'uomo artigiano. Milano: Feltrinelli, 2008).

Serres, M. (2012). Petite Poucette. Paris: Le Pommier (trad. it., Non è un mondo per vecchi. Perché i ragazzi rivoluzionano il sapere. Torino: Bollati Boringhieri, 2013). 


\section{Riassunto}

La prima parte di questo contributo è scandito su due livelli di riflessione. Il primo finalizzato a svolgere un'analisi genealogica del ruolo delle differenti tecnologie (libro e media digitali) nel ridefinire di volta in volta spazi, metodi e relazioni all'interno degli ambienti deputati all'apprendimento. Piu nel dettaglio si tratta: (a) di tratteggiare il rapporto tra l'evoluzione delle tecnologie didattiche e la necessità di sviluppare strategie di apprendimentol insegnamento compatibili all'interno di spazi adeguati; (b) di comprendere la stretta interrelazione (culturale e operativa) tra i media digitali, le nuove metodologie didattiche (di impronta costruttivista) e i differenti setting di apprendimento. Sulla base di tali premesse si procederà, pertanto, a un'analisi dei "nuovi spazi» della didattica aumentata digitalmente. In particolare, verrà messo in rilievo come l'innovazione metodologico-didattica sta co-evolvendo con l'affermarsi anche nella scuola dei media digitali; e come sia inestricabilmente connessa alla concettualizzazione e alla progettazione di nuovi «spazi» e ambienti multipli e tematizzati, dove praticare concretamente le nuove metodologie dell'apprendimento "aumentato" dalle tecnologie. La classe tradizionale è da questo punto di vista ormai superata.

Parole chiave: Ambienti di apprendimento, Apprendimento aumentato dalle tecnologie, Apprendimento cooperativo, Aula, Classe ribaltata.

How to cite this Paper: Ferri, P., \& Moriggi, S. (2016). Destrutturare l'aula, ma con metodo: spazi e orizzonti epistemologici per una didattica aumentata dalle tecnologie [Deconstruct the classroom, but with method: Spaces and epistemological horizons for a didactics implemented by technologies]. Journal of Educational, Cultural and Psychological Studies, 13, 143-161. doi: 10.7358/ ecps-2016-013-ferr 